\title{
Algunas consideraciones sobre la influencia de la heterngeneidad demográfica en el consumo y la calidad del medio ambiente
}

\author{
Haydea Izazola \\ El Colegio Mexiquense
}

Resumen

Un argumento frecuentemente socorrido al estudiar la interrelación entre población y medio ambiente, vincula el acelerado crecimiento de la población, especialmente en los países en desarrollo al deterioro ambiental planetario, sin abundar en la heterogeneidad demográfica, social, económica y cultural de la población. En consecuencia, una intervención fundamental para proteger el medio ambiente global consistiría en reforzar los programas de planificación familiar en los países pobres, sin enfrentar otros aspectos igualmente importantes que afectan la calidad ambiental.

En el presente articulo se resalta la importancia que la diversidad demográfica puede tener en la calidad del medio ambiente, a través de la diversidad de patrones de consumo determinados económica, social, política, y culturalmente.

Al revisar algunas propuestas teóricas y metodológicas para el estudio de la población en general y de su interrelación con el medio ambiente, 
en particular, se sugiere prestar especial atención a la influencia que factores como la distribución espacial de la población, su estructura por edades y género, su organización en hogares, el ciclo vital familiar, asi comosu diferenciación económica, pueden tener en la calidad ambiental tanto a nivel local como global, al incidir en los mecanismos de acceso, uso y control de los recursos naturales y en consecuencia, en la diversidad de patrones de consumo de la población.

\section{Introducción}

El tema de las interrelaciones entre población y medio ambiente ha sido relativamente poco estudiado, dada la complejidad que representa y la amplia gama de dimensiones que su adecuado conocimiento debe incorporar. Adicionalmente, las explicaciones en torno a esta interrelación han estado permeadas, con frecuencia, de argumentos neomalthusianos, que pecando de simplismo, atribuyen al mero incremento de la población el deterioro del medio ambiente. Argumentos similares vinculan el fenómeno de la pobreza al incremento acelerado de la población, en especial en el mundo en desarrollo, y en consecuencia lo enarbolan como la causa principal del deterioro ambiental.

Este tipo de razonamientos ha influenciado el conocimiento de la relación entre población y medio ambiente, así como algunas propuestas para evitar el deterioro ambiental, hasta que en los últimos años, la confluencia de especialistas de diversas disciplinas en el contexto de la organización de la Cumbre de la Tierra, celebrada en Rio de Janeiro en 1992, incluyó perspectivas de análisis que llamaban a superar la visión de una relación lineal y directa, además de la necesidad de incorporar nuevos elementos, como las escalas temporales y espaciales de los fenómenos naturales, demográficos, sociales y económicos; la influencia en el medio ambiente de la diversidad demográfica, social, cultural, económica y política de la población humana, y viceversa; el papel de los mecanismos institucionales de acceso, uso y control de los recursos naturales; así como diversos aspectos vinculados a la percepción del entorno de la población, sus actitudes y comportamientos respecto al medio ambiente; y muchos otros más (Arizpe, Paz y Velázquez, 1993 y
Arizpe, Stone y Major, 1994; Hogan, 1993; Martine, 1993; Heilig, 1993; Leff, 1993; Wood, 1993; ONU, 1994; Tudela, 1995; Izazola y Lerner, 1993; Marquette y Bilsborrow, 1994; etcétera).

Ante el gran reto que representa la incorporación de las distintas dimensiones en el análisis de la relación, se han propuesto diversos marcos teóricos alternativos a la relación lineal y directa entre tamaño de la población y calidad del ambiente, entre los que destacan el enfoque multiplicador -conocido como IPAT, por la ecuación que relaciona el impacto ambiental con el tamaño de la población, el ingreso y la tecnología (Ehrlich y Holdren, 1971 y 1974; Commoner, 1994) - la perspectiva de sistemas complejos (Tudela, 1995; García, 1986), así como la de las mediaciones entre ambos temas de estudio (Bilsborrow, 1993 y 1994; Mc Nicoll, 1994). Esto representa un llamado a la necesidad de estudiar la relación desde una perspectiva holística e interdisciplinaria que integre los aspectos mencionados, con el fin de dar cuenta de los diversos fenómenos y procesos inmersos en la amplia red de relaciones entre la dinámica demográfica y la calidad del medio ambiente.

En este sentido, con este artículo se busca contribuir a la comprensión de la relación ambiente-población, desde la perspectiva de las mediaciones, por medio de una propuesta de análisis que incorpore, por un lado, los patrones de consumo de la población, y por el otro, las distintas variables y categorías empleadas en los estudios de población con la esperanza de continuar en la línea de superar la noción a menudo presente en investigaciones sobre la interrelación entre población y medio ambiente, dominada por una perspectiva de grandes agregados de individuos, sin considerar su diversidad demográfica, económica, social y cultural. Frecuentemente se privilegian aspectos generales como el tamaño total, si acaso su densidad y, con mayor énfasis, su ritmo de crecimiento. No es que estos indicadores no sean importantes para una primera aproximación a la interrelación, sin embargo, resultan insuficientes si buscamos avanzar en el conocimiento de la misma, así como contribuir a que este conocimiento sea utilizado posteriormente como insumo para el diseño y ejecución de políticas que busquen una armonía entre la calidad ambiental y la dinámica demográfica.

A continuación se presentan algunos antecedentes del interés por el estudio de la relación entre población y medio ambiente, su evolución desde los estudios de población, para posteriormente analizar la contribución que desde éstos podemos hacer al conocimiento de la compleja relación entre población y medio ambiente, privilegiando la dimensión de los patrones de consumo y 
algunas variables demográficas que al parecer no han merecido atención suficiente en la literatura producida en México sobre el tema.

\section{Algunos antecedentes}

La preocupación por el medio ambiente y su relación con la población

La creciente preocupación por el deterioro ambiental planetario, motivada principalmente por el cada vez mayor conocimiento de los problemas ambientales globales tales como la reducción del tamaño de la capa de ozono, el calentamiento de la atmósfera, o la pérdida de biodiversidad, por mencionar algunos, ha alertado acerca de la importancia del papel que jugará en la propia sobrevivencia de la especie humana el acelerado crecimiento demográfico mundial registrado en especial durante el presente siglo.

Esta visión alarmista es en parte justificada si consideramos el acelerado crecimiento de la población experimentadodurante las últimas décadas. De acuerdo con la Organización de las Naciones Unidas, tomó a la especie humana desde su aparición sobre la Tierra hasta el año 1804 de nuestra era alcanzar los primeros 1,000 millones de habitantes, habiéndose reducido en la presente década a tan sólo 11 años el lapso necesario para agregar una cantidad similar de habitantes (ver cuadro 1).

Este crecimiento explosivo ha sido consecuencia de los desarrollos que en todos los ámbitos de la sociedad han propiciado un alargamiento de la vida, en especial al haberse asegurado el abastecimiento de alimentos y el control de enfermedades infecciosas, gracias a mejores condiciones de vivienda y salud, que han suscitado que además de vivir más tiempo, las generaciones actuales convivan con sus antecesoras y sus descendientes, generando mayor presión sobre los recursos limitados del planeta.

Sin embargo, tales desarrollos no han sido homogéneos. A nivel mundial, la esperanza de vida alcanzó los 62 años en promedio durante el periodo 1990-1995; mientras que en los países más industrializados este indicador ascendió a 74 años, en los de menor desarrollo sólo fue de 50 años (en México fue de 71). La tasa de mortalidad infantil, que comúnmente se utiliza como indicador de bienestar y desarrollo, muestra igualmente variaciones importantes: 62 defunciones por cada mil nacidos vivos a nivel mundial, con un
Cuadro 1

Población mundial desde 1804

\begin{tabular}{|c|c|c|}
\hline $\begin{array}{c}\text { Población } \\
\text { (millones) }\end{array}$ & $\begin{array}{c}\text { Año } \\
\text { calendario }\end{array}$ & $\begin{array}{c}\text { Años } \\
\text { transcurridos }\end{array}$ \\
\hline 1,000 & 1804 & \\
2,000 & 1927 & 123 \\
3,000 & 1960 & 33 \\
4,000 & 1974 & 14 \\
5,000 & 1987 & 13 \\
6,000 & 1998 & $11^{*}$ \\
\hline
\end{tabular}

*Proyección

Fuente: ONU, División de Población, Departamento de Información Económicay Sacial, World population growth from year 0 to stabilization, Nueva York, ONU, 6 de julio de 1994 (mimeo.).

mínimo de 11 en los países desarrollados y un máximo de 109 en los menos desarrollados (en México fue de 34 para el mismo periodo) (ONU, 1996).

El fenómeno de la mortalidad iunto con la fecundidad son los principales componentes del crecimiento demográfico. Al igual que en los indicadores de mortalidad, la fecundidad muestra importantes diferencias según los países. Si bien se estimaba que para el periodo 1990-1995 la tasa global de fecundidad mundial ascendía a 3 hijos por mujer, en los países desarrollados las mujeres en edad fértil tenían en promedio 1.7 hijos, y en los menos desarrollados, 5.5 (en México, 3.1). El efecto conjunto de ambos fenómenos se refleja en las tasas de crecimiento demográfico, que ascendieron en promedio a $1.5,0.4$ y $\mathbf{2 . 6 \%}$ respectivamente (1.8\% para el caso de México) (ver cuadro 2).

¿Cuál es la importancia de estos indicadores para el tema de nuestro artículo, específicamente para las interrelaciones entre población y medio ambiente?

En primer lugar, que el vertiginoso incremento de la población durante el último siglo ha promovido una utilización más intensiva de los recursos disponibles del planeta, acompañado de niveles de deterioro ambiental igualmente importantes. En un primer análisis encontraríamos una causalidad directa entre crecimiento demográfico y deterioro ambiental, y como consecuen- 
Cuadro 2

Principales indicadores demográficos

1990-1995

\begin{tabular}{|l|c|c|c|c|}
\hline Región/país & $\begin{array}{c}\text { Tasa de } \\
\text { crecimiento } \\
\text { anual } \\
(\%)\end{array}$ & $\begin{array}{c}\text { Esperanza } \\
\text { de vida } \\
\text { (años) }\end{array}$ & $\begin{array}{c}\text { Tasa de } \\
\text { mortalidad } \\
\text { infantil } \\
\text { (por cada } \\
1,000)\end{array}$ & $\begin{array}{c}\text { Tasa } \\
\text { global de } \\
\text { fecundidad } \\
\text { (hijos) }\end{array}$ \\
\hline $\begin{array}{l}\text { Total } \\
\text { mundial }\end{array}$ & 1.5 & 64 & 62 & 3 \\
\hline $\begin{array}{l}\text { Países } \\
\text { desarrollados }\end{array}$ & $\mathbf{0 . 4}$ & 74 & 11 & 1.7 \\
\hline $\begin{array}{l}\text { Países en vías } \\
\text { de desarrollo }\end{array}$ & 1.8 & 62 & 68 & 3.3 \\
\hline $\begin{array}{l}\text { Menos } \\
\text { desarrollados }\end{array}$ & 2.6 & 50 & 109 & 1.5 \\
\hline México & 1.8 & 71 & 34 & 3.1 \\
\hline
\end{tabular}

Fuente: ONU, 1996, ST/ESA/SER.A/159.

cia, a efecto de revertir este último, sería indispensable disminuir el crecimiento de la población mediante la limitación de los nacimientos.

Esta ha sido la medida que los diversos países del mundo en desarrollo han adoptado, especialmente a raíz de los compromisos contraídos en la Conferencia Mundial de Población de Bucarest en 1974. Cuantiosos recursos se han destinado para programas de planificación familiar en diversos países como medida indispensable para superar el subdesarrollo y proteger el medio ambiente, y las políticas explícitas han llegado incluso a ser de un carácter totalitario y coercitivo, como en el caso de China No obstante, ni los problemas ambientales, como tampoco los del desarrollo, entendido éste como la mejoría de la calidad de vida de la población, pueden enfrentarse mediante el exclusivo control del crecimiento demográfico.

A pesar de ello, una recomendación frecuente de organismos internacionales continúa siendo el reforzamiento de los programas de planificación familiar y de salud reproductiva,especialmente entre los grupos más pobres de la población, a fin de disminuir el deterioro ambiental. Tal preocupación por la influencia del crecimiento demográfico en el deterioro ambiental se refleja en las líneas prioritarias de investigación demográfica, destacando en la actualidad la concerniente a salud reproductiva, de preferencia entre grupos de menores ingresos.

\section{Los estudios de población}

En la década de los sesenta, cuando se registraban las más altas tasas de crecimiento en México y otros países latinoamericanos, la investigación sobre la dinámica de la población recibió la influencia directa de la teoría de la modernización, que en términos generales suponía que el paso de una sociedad tradicional a una sociedad moderna como resultado de procesos sociales y económicos vinculados a la urbanización e industrialización, traería aparejados cambios demográficos, en especial la disminución de la fecundidad como consecuencia de la caída de la mortalidad, resultado a su vez de los desarrollos sanitarios y médicos que produciría la urbanización. Este cambio de un patrón de alta fecundidad y mortalidad a uno de baja fecundidad y mortalidad, pasaría por una etapa de elevado crecimiento demográfico conocido como de transición, y cuya elaboración teórica ha sido la principal contribución de los estudios de población a las teorías del cambio social.

No obstante, la correspondencia de eșta teoría con situaciones históricas de los países desarrollados y la insuficiencia para dar cuenta de las diversas situaciones que se registraban en los países en desarrollo, favorecieron la propuesta de marcos teóricos alternativos para estudiar los fenómenos demográficos en los países no industrializados. Así, adoptando las categorías analíticas de la teoría de la dependencia desarrollada en América Latina, el enfoque histórico-estructural advirtió acerca de la insuficiencia de la teoría evolucionista de la modernización, y reconoció la necesidad de contextualizar histórica y estructuralmente los diversos fenómenos demográficos. En especial el fenómeno de la migración rural urbana, que durante las décadas de los sesenta y setenta representaba la principal causa del acelerado crecimiento de las grandes ciudades de América Latina, recibió la atención de los estudiosos de la población y su explicación giró alrededor de las diferencias estructurales que beneficiaban el desarrollo urbano-industrialen detrimento del medio rural. 
La cada vez mayor conciencia sobre la complejidad de los fenómenos demográficos hizo importantes contribuciones a los estudios de población, al superar el enfoque de grandes agregados de individuos para introducir el estudio de las diversas mediaciones entre el individuo y la sociedad, destacando la utilización de la unidad doméstica como unidad de análisis de los mismos (Mertens, et al., 1982). En este sentido se realizaron estudios sobre las estrategias de reproducción de las unidades domésticas urbanas (García, Muñoz y Oliveira, 1982) y campesinas (Arizpe, 1980; Lerner y Quesnel, 1982; Venegas, 1983; etcétera), con énfasis en los procesos migratorios y de incorporación al mercado laboral, resaltando la importancia de las redes de apoyo, así como los conflictos y las relaciones de poder, entre y dentro de los hogares, en cuyo abordaje las perspectivas de género y del curso de vida adquirieron un papel central.

Basado en la teoría de la reproducción social, este enfoque se aplicó al estudio de otros fenómenos demográficos, como la salud y la fecundidad; se manifestó la complejidad de los mismos, así como las distintas mediaciones que operan en la dinámica demográfica y que difícilmente pueden aprehenderse desde una perspectiva meramente cuantitativa, lo que dio pie a la investigación cualitativa.

En México hemos presenciado recientemente un auge de la aplicación de métodos cualitativos a la investigación sobre fenómenos demográficos, con una serie de perspectivas que combinan ambos enfoques y distintas unidades de análisis, especialmente aquellas que vinculan escalas macro y micro de los procesos estudiados, destacando la necesidad de tomar en cuenta la heterogeneidad demográfica, social, económica, cultural o política de la población, así como las interacciones entre fenómenos demográficos, sociales, económicos, etcétera.

Estos desarrollos de los estudios de población, sin embargo, no habían sido incorporados suficientemente en las preocupaciones sobre la interrelación entre dinámica demográfica y calidad ambiental, a diferencia de otros fenómenos demográficos (fecundidad, mortalidad, morbilidad, actividad económica, migración, etcétera), pues en aquella -salvo por algunas excepciones - ha predominado el interés por los grandes agregados, resultado de la preocupación de los problemas ambientales globales, así como de la limitada participación de los especialistas en población en los estudios vinculados al ambiente.

Afortunadamente, en años recientes se han realizado importantes avances por parte de estos últimos, habiendo incorporado algunos desarrollos de la propia disciplina al estudio de su interrelación con la dimensión ambiental. Destaca la incorporación de la unidad doméstica como unidad de análisis entre procesos demográficos y calidad ambiental; como ejemplo podemos citar, específicamente: los estudios de hogares de colonos en procesos de deforestación de las selvas tropicales (Bilsborrow, 1994; Pichón, en prensa; Marquette, 1995; Sydenstrycker y de Vosti, 1993); los hogares urbanos populares y los procesos de salud-enfermedad (Martínez y Vargas, en prensa; Schteingart, 1997; etcétera); la relación entre salud, migración y medio ambiente (Hogan, 1995); la emigración de ambientes urbanos deteriorados como estrategia de sobrevivencia (Izazola y Marquette, 1995); las percepciones y la perspectiva de género en la deforestación (Arizpe, Paz y Velázquez 1993; Schmink y Wood, 1992); por mencionar algunos estudios realizados en México y otros países latinoamericanos.

Sin embargo, la diversidad conceptual y metodológica en estas investigaciones dificulta la sistematización del conocimiento desarrollado, pues como señalan Marquette y Bilsborrow (1994), el concepto de ambiente está lejos de contar con un consenso entre los, investigadores, dado que éstos lo definen en cada caso a priori y de acuerdo con sus propios intereses, variando desde la calidad y el uso de algunos recursos naturales, hasta aspectos sociales del ambiente en que se desenvuelven los grupos humanos, tales como la influencia de factores culturales, económicos y políticos en los mecanismos de acceso, uso y control de los recursos naturales. El propio concepto de deterioro tampoco cuenta con un acuerdo entre los estudiosos del tema (Landa y Carabias, en este volumen).

Por otro lado, en términos metodológicos existe igualmente una amplia variedad: desde el uso de grandes agregados de individuos y sus relaciones cuantitativas con variables ambientales mediante la aplicación de modelos estadísticos, hasta enfoques netamente cualitativos, con igual variedad de técnicas (desde entrevistas en profundidad hasta grupos focales, en los que se exploran incluso los mecanismos psicológicos de la percepción ambiental).

Esta diversidad de enfoques sin duda ha contribuido a una mejor comprensión de la interrelación entre población y medio ambiente, y ha puesto de relieve la necesidad de incorporar en su análisis las distintas mediaciones de la relación desde una perspectiva holística e interdisciplinaria. Sin embargo, algunas mediaciones de gran relevancia no han merecido suficiente atención. Referimos, entre ellas, a la dimensión de los patrones de consumo vinculados a la diversidad demográfica. 'Aproximarse al conocimiento de la 
manera en que esta mediación opera en la relación que nos ocupa requiere, al igual que el estudio de cualquier faceta de la realidad social, el concurso de distintas especialidades y la valoración de las contribuciones que otras especialidades puedan realizar a este objeto de estudio. A continuación nos centraremos principalmente en algunos acercamientos que podrían realizarse desde los estudios de la población.

Patrones de consumo y diversidad demográfica

\section{Algunas reflexiones}

A lo largo de la historia de la humanidad, la sobrevivencia de la población ha dependido de la disponibilidad de recursos naturales estratégicos como el agua, el aire y la tierra, asociada a los primeros asentamientos humanos y al surgimiento de las grandes civilizaciones. La domesticación de especies vegetales y animales, así como la generación y el control de excedentes, propiciando sociedades cada vez más complejas, cuyas necesidades fueron modificándose, dando lugar a patrones de consumo específicos.

Diversos autores han resaltado que los patrones de consumo, determinados por una serie de factores sociales, económicos, políticos, ambientales, demográficos y culturales, se encuentran en la base misma de la relación entre población y medio ambiente (Heilig, 1993 y 1995; Martine, 1993; Provencio, s/f; Engelman, 1994; De Sherbinin, 1993, etcétera), al vincular las esferas productiva y de consumo con los habitantes de un territorio determinado y sus impactos en el medio ambiente.

A raíz de la Revolución industrial y, posteriormente, con la producción a gran escala de bienes de consumo duradero, tales como el automóvil, los aparatos electrodomésticos y otros productos industrializadosy desechables - que han marcado el desarrollo de las sociedades en el presente siglo-, las necesidades de la población se han transformado de una manera radical, y la producción de sus satisfactores deviene el principal motor de la reproducción del sistema socioeconómico y de los problemas ambientales a nivel global.

La población humana se vincula al medio ambiente a través de procesos de producción y consumo asociados a la satisfacción de sus necesidades, mismas que son determinadas socialmente dependiendo del momento histórico, la estructura económica predominan-

te, la diferenciación social, las pautas culturales, etcétera, además de la disponibilidad de recursos naturales. Con el desarrollo de nuevas tecnologías, este Último factor deja de ser determinante, pues la aparición de bienes sustitutos permite una mayor oferta de ciertos bienes estratégicos para la subsistencia humana.

Durante el presente siglo hemos sido testigos de avances tecnológicos de gran envergadura, que sin embargo han afectado el equilibrio ecológico del planeta, y desde la década de los años sesenta las voces de alarma no han cesado de advertir su peligro en la subsistencia de la especie humana.

A pesar de que la relación de la población con los procesos de producción y consumo resulta determinante para poder comprender los procesos ambientales que actualmente llaman la atención de investigadores, tomadores de decisiones y sociedad en general, su análisis demanda la incorporación de diversos enfoques disciplinarios. Por un lado, la economía contribuye al dar cuenta de la estructura productiva en diversas escalas espaciales, como países, regiones, localidades, etcétera, identificando aquellos procesos productivos con mayor impacto en el medio ambiente. En los últimos años una rama de la economía se ha abocado a la dimensión ambiental y se ha propuesto una serie de mecanismos, principalmente de tipo financiero, para evitar la degradación ambiental en distintas ramas de la actividad económica, aunque no se encuentran libres de críticas por su dudosa efectividad (Leff, 1995).

Por otro lado, las ciencias políticas y la sociología alertan sobre la injusticia en la distribución de los recursos y en la toma de decisiones estratégicas para detener el deterioro ambiental. Se ha llamado la atención acerca de la influencia que importantes intereses económicos han tenido en el surgimiento de la crisis ambiental a nivel global, así como de la falta de compromiso para adoptar medidas para resolverla.

La solución a los problemas ambientales dista mucho de ser simple, y a efecto de contribuir a su comprensión, es indispensable saber cómo la población humana hace uso del medio d i e n te. Una aproximación a esta dimensión puede realizarse a través del análisis de los patrones de consumo de distintos grupos sociales, para lo cual resulta de fundamental importancia conocer sus características demográficas, en virtud de que la dinámica de las poblaciones, su estructura y su composición en hogares nos pueden proporcionar ciertas pistas acerca de la evolución futura de la demanda de ciertos recursos naturales, y en consecuencia, anticipar acciones que contribuyan a un menor deterioro ambiental. 
Por lo tanto, el hecho de pensar que las necesidades básicas de la población (alimentación, vivienda y vestido), así como su tamaño y ritmo de crecimiento, serán las variables fundamentales de la capacidad del planeta para garantizar el futuro de la especie humana, lleva a cuestionar argumentos neomalthusianos, que parecen dominar aún ciertas esferas de pensamiento científico y político.

Hace unos años, Davis (1991) aducía la necesidad de incorporar la dimensión cultural del consumo en el estudio de la interrelación entre población y recursos naturales, dada la importancia que en la sociedad actual tiene la posesión de bienes materiales. Advertía que a pesar de que el hombre había alcanzado logros importantes en materia tecnológica y científica, que podrían permitirle disfrutar de una mejor calidad de vida y un mayor tiempo libre, en la sociedad industrial se promueven actitudes y valores que alientan la acumulación de bienes materiales, convirtiendoel trabajo en un fin, más que en un medio, para alcanzar un nivel mínimo de bienestar.

Pero, jacaso toda la población puede acceder a la gran variedad de bienes materiales al alcance de las sociedades industrializadas?

Esto sería, aparentemente, el fin último de la filosofía de la globalización de los mercados; sin embargo, al igual que la diversidad de algunos indicadores demográficos ya mencionados, la población mundial presenta un mosaico de situaciones económicas, políticas, sociales y culturales, que determinan tanto los mecanismos institucionales de acceso, uso y control de los recursos naturales, como las necesidades y sus satisfactores, y en consecuencia, los patrones de consumo.

En las sociedades en las que la satisfacción de las necesidades básicas se realiza directamente a través de la explotación de los recursos naturales disponibles en el entorno inmediato, sin relaciones con mercados extrarregionales, el conocimiento de los mecanismos institucionales de acceso, uso y control de esos recursos podría resultar suficiente para determinar los patrones de consumode los individuos y hogares, los cuales corresponderían a las labores productivas desarrolladas en el nivel local, con limitados intercambios.

En las sociedades cuya reproducción social y materia depende principalmente del mercado, la disponibilidad de medios económicos representa un factor fundamental para acceder a los satisfactores que definen los patrones de consumo. En tal virtud, una dimensión central para aproximarnos a la estructura del consumo consistiría en analizar el ingreso de los hogares y sus determinantes, como por ejemplo, la inserción ocupacional de sus miembroseconómicamente activos y su escolaridad.

En ambos casos las características demográficas, tanto de individuos como de hogares, contribuyen a una mejor comprensión de las relaciones señaladas. Destacan la distinción entre localidades rurales y urbanas, la estructura por edades y sexo de los individuos y la estructura de los hogares, debido a la influencia que pueden tener para entender la dinámica del consumo y sus posibles efectos en el medio ambiente.

\section{La dimensión espacial}

Existe una amplia gama de acercamientos a la dimensión espacial de los fenómenos demográficos. Un corte freçentemente socorrido y que ha probado amplia eficiencia en la explicación de los mismos, lo representa la división rural-urbana, cuya definición se enfrenta a múltiples problemas.

Por ejemplo, en términos exclusivos del tamaño de la población, no existe consenso acerca del límite ideal para caracterizar a una localidad como rural o urbana. La definición censal en México consiste en considerar rurales a aquellas localidades de menos de 2,500 habitantes, y como urbanas a las que superen este número. Algunos estudiosos de lo urbano han sugerido un corte de 15,000 habitantes, mientras que algunas recomendaciones internacionales sitúan este límite en 20,000. Esta diversidad de definiciones nos muestra la dificultad para determinar con base en un solo indicador la complejidad de la dimensión espacial de la realidad.

Lo mismo sucede si privilegiamos en la definición de ruralidad el predominio de las actividades económicas primarias, pues puede darse el caso de que en comunidades relativamente apartadas y con poca población, la actividad económica preponderante no sea exclusivamente del sector primario, invalidando el criterio basado en la exclusiva actividad económica.

Por otro lado, la disponibilidad de servicios urbanos es otro de los criterios empleados para diferenciar a las comunidades rurale de las urbanas; sin embargo, en especial en sociedades industrializadas, localidades poco comunicadas y con un pequeño número de habitantes disponen de toda la infraestructura considerada como "urbana", a saber: agua potable, alcantarillado, escuelas, centros de salud, etcétera. 
La dificultad de considerar un criterio único para determinar la situación rural o urbana de las comunidades, nos lieva a proponer con fines analíticos un continuum, en uno de cuyos extremos podríamos ubicar a grupos rurales basados en la explotación de los recursos naturales de su entorno inmediato, con poca o nula influencia de agentes externos para la satisfacción de sus necesidades, y en el otro, a las megaciudades, en las que dicha satisfacción, si bien basada en la explotación de recursos naturales, se encuentra mediada por múltiples factores entre los que destaca el mercado extrarregional.

Como se mencionó con anterioridad, en las sociedades cuya reproducción se basa en la explotación y autoconsumo de los recursos naturales de su entorno inmediato, el estudio de la relación entre población y medio ambiente puede resultar menos complicado al poderseidentificar directamentelos mecanismos institucionales de acceso, uso y control de los recursos que definen los patrones de consumo, la influencia de la dinámica demográfica en los mismos, así como en procesos de conservación o deterioro ambiental, además del desarrollo de mécanismos de adaptación a posiblesdesequilibrios ecológicos.

Así, podría determinarse con relativa facilidad la manera en que el crecimiento de la población afecta la capacidad de carga de los ecosistemas locales, y en caso de desequilibrios que pusieran en riesgo la sobrevivencia del grupo estudiado, los mecanismos de adaptación a los que se recurriría; entre éstos, la intensificación de los procesos productivos, la reducción del consumo de bienes escasos, la disminución de la fecundidad, las modificaciones en la estructura de parentesco y de los hogares, o la emigración.

En un texto clásico, Boserup (1965) examina la capacidad de los grupos humanos para hacer frente a los retos que impone el crecimiento de la población, gracias al desarrollo de tecnologías más intensivas de producción. Por su parte, Meggers (1976) señala los mecanismos para controlar el crecimiento de la población, a los que recurren algunos grupos amazónicos. Otros estudiosos de la relación han privilegiado el medio rural, por la relativa "transparencia" de las relaciones estudiadas.

No obstante, cabe mencionar que en la actualidad cada vez son más escasos los grupos sociales que dependen para su reproducción de la exclusiva explotación y autoconsumo de su entorno inmediato, dada la creciente integración al mercado y la monetarización de las actividades propias del medio rural.

En cuanto a los niveles de consumo, podríamos suponer que la explotación de los recursos naturales responde, en este tipo de comunidades, principalmente a la satisfacción de necesidades básicas: alimentación, vestido, vivienda y salud; los patrones de consumo se determinan con base en estas necesidades.

La situación se torna compleja al abordar la relación población-ambiente en sociedades más desarrolladas, y al llegar al otro extremo del continuum: las grandes ciudades de la actualidad, en donde la satisfacción de las necesidades se realiza principalmente por medio del mercado, y en donde la relación con el entorno natural - bastante directa entre los grupos rurales dedicados a actividades primarias - se ha transformado en una relación indirecta, dado el predominio creciente de un ambiente artificial y con nuevas necesidades satisfechas principalmente por medio del mercado, que se alejan cada vez más de los requerimientos básicos de alimentación $\mathrm{y}$ vivienda.

\section{La betenogeneidad económica}

En la discusión acerca del impacto de la población en la calidad del ambiente, se ha documentado la influencia que tiene la ubicación de los individuos en la estructura económica con respecto al acceso a los recursos naturales y a la calidad de los mismos. Se ha mencionado que la responsabilidad del deterioro ambiental se puede identificar con los patrones de consumo de la porción más rica de la humanidad, cuya capacidad económica garantizael acceso a bienes materiales que tienen un grave impacto en el medio planetario. Por su parte, se argumenta que la porción más pobre de la población mundial, dado su limitado ingreso y su ritmo de crecimiento, utiliza de manera menos sustentable los recursos naturales a su alcance, especialmente en zonas ecológicamente vulnerables como las selvas tropicales.

A efecto de aproximarnos a esta diversidad, una primera tarea consistiría en caracterizar a la población que interesa d a $\mathrm{r}$, en un momento y espacio determinados, de acuerdo consu posición en la estructura social y económica, determinada por distintos atributos. Un primer criterio podría ser el ingreso de los individuos y sus hogares, como mecanismo fundamental de acceso a los recursos naturales en sociedades monetarizadas, mediante la observación de sus patrones de consumo. Sin embargo, no todos los grupos de población satisfacen sus necesidades y consumen por 
medio del mercado, por lo que es necesario contemplar otros atributos -especialmente en comunidades rurales de países en desarrollo-, tales como su actividad económica que, como antes se señaló, coincide con algunos aspectos de su relación con los recursos naturales y el ambiente. La ocupación y el sector de actividad pueden resultar útiles para caracterizar la diversidad económica y social, pero también como aproximación a su relación con el medio. Es posible hacer esta caracterización a nivel de grandes agregados de individuos, aunque el análisis se enriquecería al utilizar las unidades domésticas, definiéndolas con las características económicas del jefe del hogar, pero preferentemente de todos los miembros económicamente activos. Otra posibilidad es observar la distribución del ingreso de los hogares, con la típica clasificación por deciles de ingreso.

Una vez caracterizada la población en estudio, habría que clasificar los patrones de consumo de los distintos grupos sociales, a distintas escalas espaciales y temporales, con el fin de documentar su evolución en el tiempo, dando prioridad a aquellos bienes "críticos" por su impacto en la calidad del ambiente (Provencio, s/f).

Fuentes de información disponibles como las encuestas de ingresos y gastos - levantadas en una gran cantidad de paísespodrían facilitar la comparación internacional, así como dentro de los países. No se trata de fuentes perfectas, pero podrían representar la base para posteriormente profundizar el análisis con técnicas más depuradas. Este tipo de encuestas proporciona información detallada de muestras representativas a nivel nacional de los hogares, tanto del ingreso como del gasto, con un minucioso desglose que favorece la aproximación a los patrones de consumo de los diversos grupos sociales.

Mediante el análisis de esta información se podrían identificar tanto los bienes consumidos por diversos grupos económicos, como su impacto en el ambiente. Si bien está documentado que durante los últimos años la distribución del ingreso en nuestro país se ha vuelto más desigual y que las diferencias se han ensanchado, podemos suponer que los grupos menos privilegiados tendrían un consumo mucho menos impactante en términos ambientales, por la sencilla razón de que su propia situación de miseria les impide acceder a aquellos bienes con un grave impacto en el medio ambiente, especialmente de manera global (vehículos automotores, aparatos domésticos y productos desechables, entre otros bienes industrializados).
La estructura del consumo de los grupos menos privilegiados económicamente se encuentra dominada por aquellos bienes destinados a la satisfacción de las necesidades básicas, pero principalmente a la alimentación de los miembros del hogar. No obstante, su impacto en la calidad de los recursos naturales podría resultar muy importante dada su magnitud y ritmo de crecimiento, especialmente en ecosistemas frágiles como las selvas tropicales, y en los cinturones de pobreza que rodean las zonas urbanas.

En virtud de que la información que proporcionan las encuestas de ingresos y gastos proviene de muestras representativas a nivel nacional de los hogares a lo largo del tiempo, se puede contar con una primera aproximación a los patrones de consumo de los distintos grupos de población, aunque se trata de información sobre el gasto de los hogares, lo cual no necesariamente abarca todo el consumo y su complejidad, en especial de aquellos mecanismos de acceso a los recursos no monetarizados.

En tal virtud, es indispensable combinar este tipo de fuentes con otras vías de recolección de información, entre las que destacan los métodos cualitativos, que nos permiten acercarnos al significado del consumo entre los diversos grupos económicos de población, a los mecanismos de acceso a los recursos naturales, y al impacto real de conductas consumistas en el entorno. Empero, la riqueza del análisis no radica exclusivamente en esta dimensión económica; es indispensable incorporar las características demográficas de la población estudiada.

\section{La beterogeneidad demográfica}

Además de la heterogeneidad económica de la población, que representa un mecanismo fundamental del acceso a los recursos naturales, y en consecuencia, de los patrones de consumo, existe una serie de variables demográficas que convendría tomar en cuenta al abordar esta mediación. Comenzaremos por el tamaño de la población; posteriormente nos centraremos en la dinámica demográfica, acentuando las modificaciones en la estructura de la población, tanto por edad como por su organización en hogares, proponiendo algunas hipótesis acerca de su posible influencia en la relación con el ambiente, mediante las modificaciones que podrían originar en los patrones de consumo. 
Tamaño de la población

El propio tamaño de una población podría representar el elemento demográfico central de la mediación a que hemos hecho referencia. Al menos cada habitante del planeta debería contar con una serie de recursos mínimos que le permitieran alimentarse adecuadamente y contar con una vivienda de condiciones mínimas. Pero esta "utopía" ha sido materia de amplios debates y conflictos desde tiempos remotos, ante las desigualdades sociales y económicas que han caracterizado la historia de la humanidad.

Ya hemos presentado algunas cifras del crecimiento de la población mundial y cómo en el presente siglo se han alcanzado niveles nunca antes registrados, con importantes variaciones entre países desarrollados y en vías de desarrollo (ver supra).

Si supusiéramos que las necesidades básicas de la población determinan su consumo y en consecuencia su impacto en la explotación de los recursos naturales, cerca de 6,000 millones de habitantes deberían: consumir al menos 2,500 calorías al día; contar con un espacio mínimo para refugiarse de las inclemencias del clima; disponer de un consumo mínimo de 50 litros de agua al día; esto por mencionar las necesidades más elementales. Podemos imaginarnos la carga que sobre los recursos naturales imprime esta magnitud de población, aspecto que ha sido motivo de advertencias con respecto a los límites del crecimiento, hecho que refuerza los argumentos neomalthusianos, a pesar de que de acuerdo con diversas estimaciones (Heilig, 1993 y 1995), el planeta tendría una capacidad casi ilimitada para hacer frente a las crecientes demandas de una población en expansión.

Sin embargo, como señalamos en relación con los fenómenos demográficos, las desigualdades entre países y dentro de los mismos, nos alertan acerca de la diversidad de cargas ambientales de las distintas sociedades, y es precisamente en esta diversidad demográfica que nos centraremos a continuación, en un intento por establecer algunas relaciones prioritarias para comprender su papel en los patrones de consumo de la sociedad.

\section{Dinámica demográfica y estructura por edades}

Las grandes transformaciones sociales, económicas y políticas registradas desde mediados del presente siglo, han modificado radicalmente la estructura y distribución de la población. En los países en desarrollo se atraviesa en la actualidad por periodos de elevado crecimiento demográfico, situación denominada por los teóricos de los estudios de población como la etapa transicional, en la que la fecundidad disminuye posteriormente a la caída de la mortalidad, reportada como consecuencia de la aplicación de los avances médicos y sanitarios que controlan las enfermedades infecciosas. Esta etapa viene acompañada de una estructura por edades de la población bastante joven, a diferencia de aquellas poblaciones postransicionales en las que la estructura por edades se va envejeciendo.

En una población donde se registran elevadas tasas de fecundidad y mortalidad, la tasa de crecimiento demográfico es reducida y la estructura por edades permanece sin cambios importantes; sin embargo, en una población que logra controlar la mortalidad, a consecuencia de mejoras sanitarias y médicas, pero cuya fecundidad es elevada, la tasa de crecimiento aumenta y la estructura por edades muestra una base amplia, es decir, se caracteriza por un predominio de población joven. Por otro lado, en aquellas sociedades en las que disminuye la fecundidad y la mortalidad también es baja, se registra un proceso de envejecimiento de la población, dado que la renovación de generaciones es muy lenta. Este sería el caso de las poblaciones de países desarrollados, en los que la pirámide de edades cambia de forma, ensanchándose en las edades adultas. La población mexicana, que registró una importante caída de la fecundidad desde la década de los años setenta, comienza a ensancharse en edades menores de 30 años (ver gráfica 1).

La estructura por edades de la población incide en muchos aspectos de la vida-social y económica; y en consecuencia sobre el medio ambiente, al ejercer presión sobre ciertos recursos estratégicos. En una sociedad con una estructura por edades joven, resultado de altos índices de fecundidad y bajos de mortalidad, con tasas de crecimiento elevadas, este ritmo hace que resulten insuficientes las inversiones para garantizar suficiente alimentación, educación básica, infraestructura sanitaria y atención primaria a la salud, condiciones agravadas por la propia situación de dependencia de los países en desarrollo, así como por los procesos de urbanización e industrializaciónque promueven la migración ruralurbana, la expansión territorial de las zonas urbanas y los consecuentes impactos ambientales frente a una inadecuada infraestructura. Las grandes ciudades de los países en desarrollo son claros ejemplos de esta situación. 

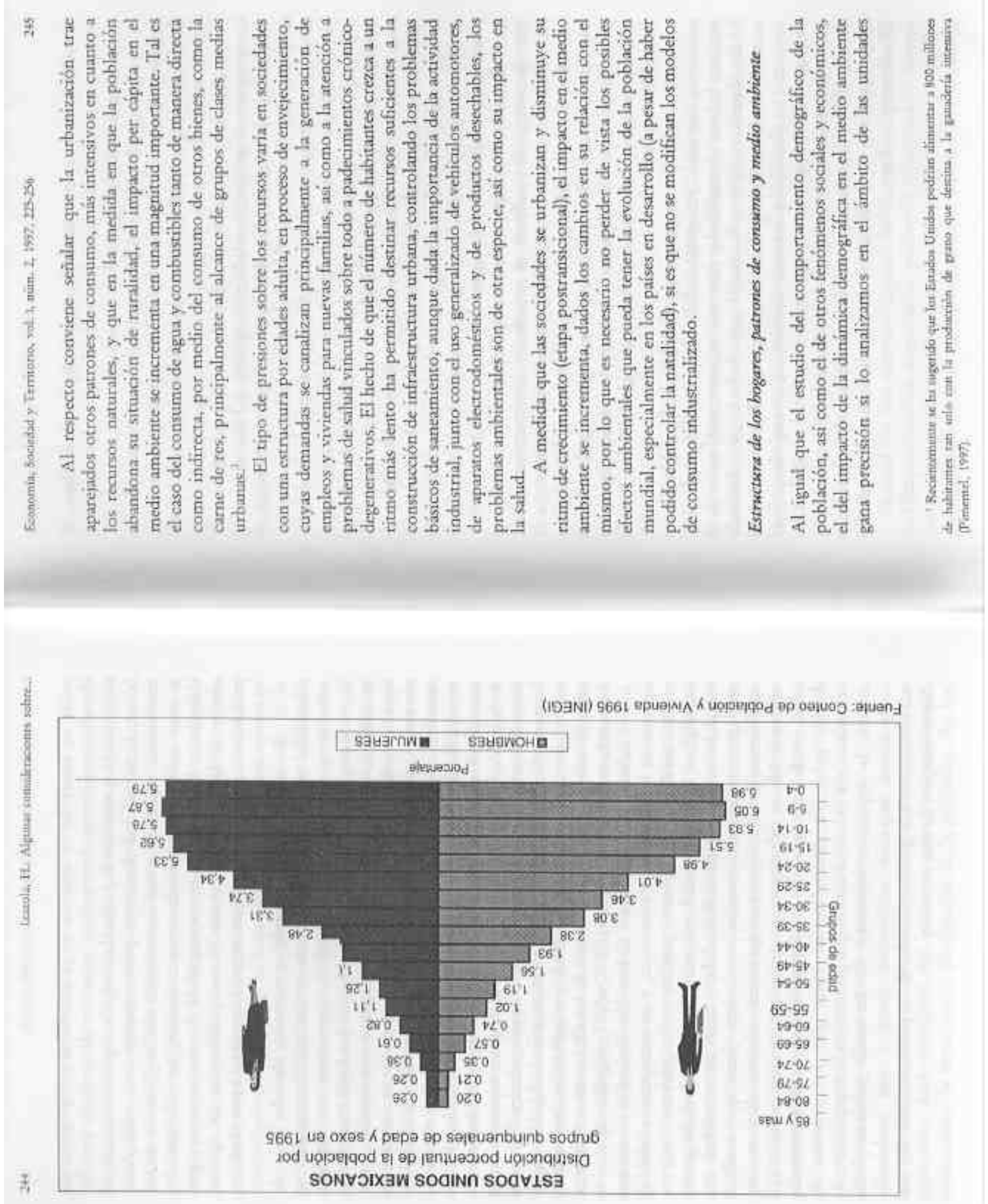
domésticas en que se encuentran organizados los individuos, al representar una instancia mediadora entre el acceso a los recursos y los consumos individual y social.

La consideración de la composición y estructura de los hogares, así como el ciclo de vida familiar, constituyen elementos fundamentales para aproximarnos a la mediación a que nos hemos venido refiriendo.

En las sociedades desarrolladas, con un importante componente de población adulta y/o anciana y bajos niveles de fecundidad, la organización familiar en hogares pequeños y unipersonales tiene una influencia distinta sobre los recursos naturales y la ocupación del espacio físico que afecta su relación con el ambiente, dadas sus necesidades básicas socialmente creadas, que superan la mera sobrevivencia y buscan mejor calidad de vida con confort y un mayor espacio habitable.

La necesidad de un mayor número de viviendas, independientemente de la tasa de crecimiento de la población, hace necesaria la ampliación del suelo urbano, a costa de la tierra destinada a actividades agropecuarias. La consiguiente construcción de infraestructura de comunicaciones -especialmente para autotransporteque cubre de material impermeable amplias superficies estratégicas para la recarga de acuíferos, es una de las consecuencias que la estructura por edades, vinculada a patrones reproductivos y a la estructura de los hogares, puede tener en la esfera ambiental. Esta creciente demanda de suelo urbano se registra igualmente en poblaciones con un elevado ritmo de crecimiento, aunque las causas son ciertamente distintas, con frecuencia identificadas con la migración rural-urbana.

En tanto la estructura por edades de la población se encuentre dominada por grupos adultos, aún en edad productiva, con una limitada fecundidad y ante una constante ampliación de su poder adquisitivo, la proporción del gasto de los hogares destinada a cubrir las necesidades básicas disminuye en la estructura del consumo. La adquisición de enseres domésticos, automóviles y viajes de placer cada vez más ostentosos explica en parte el por qué en los países desarrollados - no obstante haber controlado su crecimiento demográfico- el impacto que cada habitante tiene sobre el medio es muy superior al de un habitante de un país en vías de desarrollo (Wackernagel y Rees, 1996).

\section{La importancia del género y el ciclo vital}

Por su parte, el menor tamaño de los hogares se encuentra asociado a la jefatura femenina y a la mayor escolaridad de las mujeres, así como a su participación en la actividad económica; de ahí la importancia que recientemente se le ha dado en las políticas de población al empoderamiento (empowerment) de las mujeres.

En los países industrializados, ante la importancia que el mercado tiene en la reproducción cotidiana de los hogares, es indispensable considerar la participación femenina en el mercado laboral, su posible influencia en los patrones de consumo y su consecuente impacto ambiental. Generalmente la participación de las mujeres en la actividad económica contribuye a la adquisición y al mejoramiento de la infraestructura doméstica, pero el peso que representa para ellas la doble jornada (siendo económicamente activas), las vuelve más dependientes de bienes industrializados para la reproducción cotidiana de sus unidades domésticas. La oferta de alimentos industrializados (congelados o enlatados), así como de productos desechables, detergentes y material de limpieza doméstica. de alta eficiencia, con frecuencia elaborados con compuestos químicos de alta toxicidad, tienen un impacto importante en el ambiente, tanto por la generación de desechos domésticos como por la contaminación de los cuerpos de agua a los que son arrojados. Sin embargo, representan insumos indispensables en casi todos los hogares, y su utilización se intensifica en la medida en que las mujeres -también principales encargadas de la reproducción cotidiana de los hogares industrializados- se incorporan al mercado laboral.

La organización en hogares pequeños y unipersonales por parte de la población de las sociedades desarrolladas, propicia que se incurra en deseconomías de escala en el ámbito de las unidades domésticas, tanto por lo que respecta al uso y aprovechamiento del suelo, la energía, los alimentos y el agua, como de los enseres domésticos, por mencionar los más obvios.

El hecho de que en los países en desarrollo se registren elevados tamaños promedio de los hogares, aun con una proporción importante de estructuras no nucleares -muchas veces en condiciones de hacinamiento-, obliga a hacer un uso más eficiente y racional de los recursos disponibles. La propia situación de pobreza de amplias capas de la población refuerza este manejo menos derrochador, tanto del espacio como de los recursos naturales estratégicos, a pesar de que comúnmente se atribuye a la condición 
de pobreza de la población de las sociedades en desarrollo, el deterioro ambiental global.

Aproximarnos a la relación entre estructura y composición del hogar y patrones de consumo, es posible por medio del análisis de las encuestas de ingresos y gastos de los hogares, no obstante las limitaciones señaladas. La información en ellas contenida a propósito de la estructura y composición de los hogares, resulta de suma utilidad como punto de partida hacia análisis más profundos. En este sentido, convendría aproximarnos a las estrategias de sobrevivencia de los hogares desde una perspectiva ambiental, mediante métodos cualitativos, por ejemplo, distinguiendo la composición de los hogares y privilegiando aquellos dirigidos por mujeres, a menudolos más pobres entre los pobres, a efecto de poder conocer el impacto ambiental que tienen sus patrones de consumo precario y su insuficiente acceso a la infraestructura urbana, especialmente en los cinturones de miseria de las grandes ciudades, coadyuvantes en importantes problemas ambientales.

Adicionalmente, convendría tomar en cuenta las fases del ciclo vital familiar en la conformación de los patrones de consumo y su impacto en el medio. Por ejemplo, en las sociedades industrializadas a medida que la estructura por edades se envejece, su organización en hogares predominantemente unipersonales, además de su perfil epidemiológico, propician que en edades avanzadas la población se enfrente a nuevas necesidades que afectarán su patrón de consumo. Es posible que en la medida en que se requiera de la ayuda de otras personas para su mantenimiento cotidiano, y ante la oferta de atención que dan las instituciones de seguridad social de países desarrollados, se recurra a instituciones como asilos y hospitales, con impacto diferencial sobre el ambiente. En este sentido, ¿cuál podría ser el impacto ambiental de la modificación de los patrones de consumo? Por un lado, la vivienda colectiva podría llevar a un uso más eficiente tanto del espacio como de los energéticos y los alimentos; por el otro, la creciente dependencia de medicamentos y las estancias hospitalarias podrían modificar la composición de los desechos y la basura municipales, pero también comprometerían la utilización y el control de determinados recursos naturales indispensables para la producción de medicamentos y la elaboración de dietas menos nocivas para el organismo humano.

Por su parte, en las sociedades en vías de desarrollo la atención de personas de edad avanzada recae en sus familiares, dada la limitada seguridad social institucionalizada con que se cuenta, propiciando la extensión de los hogares, con la consecuente utilización más intensiva de los recursos y del espacio. Sin embargo, las transformaciones sociales y económicas que han afectado el sustento de las redes de apoyo y solidaridad dentro de los hogares, pueden promover la creación de hogares unipersonales en fases avanzadas del ciclo vital, que -en ausencia o insuficiencia de pensiones de vejez- podrían darse en condiciones de extrema pobreza. Su posible impacto, no sólo en el ambiente, sino en la calidad de vida de la población de la tercera edad, deberá ser materia de investigación futura.

El impacto ambiental de los patrones de consumo es muy distinto en fases tempranas del ciclo vital entre sociedades industrializadas y no industrializadas; en estas últimas, el acceso a los servicios mínimos de saneamiento es muy desventajoso. El impacto de un recién nacido en una ciudad como Boston, por ejemplo, definitivamente es muy superior al de un bebé en Lagos.

Es indispensable considerar cómo todos estos elementos pueden afectar el acceso a los recursos, los patrones de consumo y su impacto en el medio ambiente, y no abordar esta cuestión exclusivamente como si todos los humanos tuviéramos el mismo acceso a los recursos y, en consecuencia, una misma relación con nuestro entorno.

\section{Conclusiones}

El estudio de la relación entre población y medio ambiente privilegiando la mediación de los patrones de consumo hace indispensable la consideración simultánea de aspectos como el espacio en que vive la población, la actividad productiva, el nivel de ingreso, la distribución del mismo, las expectativas de consumo -determinadas cultural y publicitariamente-,además decaracterísticas demográficas como la edad, el sexo, la organización familiar y el momento del ciclo vital individual y familiar, aspectos todos que determinan consumos específicos.

La aproximación al impacto que puede tener la estructura por edades de la población en el ambiente por medio del consumo, así como su organización en distintos tipos de hogares, puede realizarse desde diversas perspectivas. La disponibilidadde encuestas de ingresos y gastos, levantadas en distintos momentos y paises, nos permite una primera aproximación a la influencia que la distribución del ingreso de los hogares tiene en esta relación. Sin embargo, 
información que también es captada por estos instrumentos y que no ha sido explotada con este fin, puede proporcionar pistas valiosas sobre el complejo papel que desempeñan la diversidad demográfica y la heterogeneidad de la estructura y composición de los hogares en la conformación de los patrones de consumo, vinculados a la desigualdad económica, y en cuanto al acceso a los recursos.

Como han demostrado los estudios de población orientados a la explicación de algunos fenómenos demográficos, con el fin de enriquecer el análisis es indispensable la combinación de perspectivas como las que nos pueden proporcionar estos instrumentos de amplia cobertura, con otras técnicas de acercamiento a la realidad, como los métodos cualitativos que arrojan luz sobre otros elementos centrales y que difícilmente pueden ser aprehendidos con este tipo de encuestas. Nos referimos a la riqueza que los métodos cualitativos, de corte antropológico, ofrecen para profundizar en algunos elementos que se encuentran en la base de decisiones pertinentes para las relaciones que nos interesa explicar. En el caso que nos ocupa, podríamos explorar cómo a lo largo de la vida de una persona se han modificado los mecanismos de acceso a los recursos naturales, su influencia en los patrones de consumo, y a su vez, cómo éstos han variado a lo largo del tiempo, junto con las distintas experiencias vitales de los individuos y las familias. Por ejemplo, con la llegada de nuevos miembros al hogar, su incorporación al mercado laboral, su matrimonio, migraciones, etcétera. También podríamos aproximarnos al papel que, por ejemplo, tiene la búsqueda de prestigio social en el consumo entre algunos grupos sociales, y cómo, incluso ante recursos limitados, se privilegian renglones del consumo que se alejan de la satisfacción de necesidades básicas, para adquirir prestigio social, determinado por la cultura dominante. Sobre este aspecto podemos citar el consumo de carne de res en lugar de vegetales con alto valor proteico, o bien la adquisición de autos de lujo en lugar de vivienda; aun si es sencilla; esto podría darnos ciertas pautas acerca de la importancia que la dimensión subjetiva tiene en el comportamiento de la población y su relación con el medio. Incluso puede estar en la misma base de decisiones netamente demográficas, como la migración, la nupcialidad, la fecundidad, etcétera, al vincularse crecientemente con la disponibilidad de medios para acceder a cienos recursos.

Como podemos apreciar, la diversidad de enfoques y unidades de análisis, así como de fuentes de información y herramientas analíticas para el estudio de la relación entre patrones de consumo y dinámica demográfica como mediaciones de la interrelación entre población y ambiente, prometen ser una fuente inagotable de inspiración para los investigadores interesados en este interesante campo de estudio.

\section{Bibliografía}

Arizpe, Lourdes (1980), La migración por relevos y la reproducción social del campesinado, México, El Colegio de México, Cuadernos del CES, núm. 28.

-, Fernanda Paz y Margarita Velázquez (1993), Cultura y cam bio global: Percepciones sociales sobre la deforestación en la Selva Lacandona, México, CRIM/UNAM-Miguel Ángel Porrúa.

- y Margarita Velázquez (1994), "The Social Dimensions of Population", en L. Arizpe, P. Stone y D. Major (eds.), Populationand Environrnent. Rethinking the Debate, Boulder, Colorado, Westview Press.

_, Priscilla Stone y David Major (eds.) (1994), Population and Environment. Rethinking the Debate, Boulder, Colorado, Westview Press.

Bilsborrow, Richard (1993), "Reflexiones metodológicas sobre las interrelaciones entre procesos demográficos y problemas del ambiente en áreas rurales de América Latina", en Haydea Izazola y Susana Lerner (comps.), Población y ambiente: inuevas interrogantes a viejos problemas?,México, SOMEDE-E1 Colegio de México-The Population Council.

(1994), "Population Development and Deforestation: Some Recent Evidence", en ONU, Population, Environment and Development: Proceedings of the United Expert Group Meeting on Population, Environment and Development, Nueva York, ONU.

Boserup, Ester (1965), Conditions of Agricultura1 Growth, Chicago, Aldine. 
Commoner, Barry (1994), "Population, Development and the Environment: Trends and Key Issues in the Developed Countries", en ONU, Population, Environment and Development: Proceedings of the United Expert Group Meeting on Population Environment and Development, Nueva York, ONU.

Corona, Rodolfo et al. (1986), Problemas metodológicos en la investigación sociodemográfica, México, PISPAL-El Colegio de México.

Davis, Kingsley (1991), "Population and Resources: Fact and Interpretation", en K. Davis y M. S. Bernstam (eds.) Resources, Environment and Population: Present Knowledge, Future Options, Population and Development Review. Suplemento del vol. 16, 1990, The Population Council.

De Sherbinin, Alex (1993), Population and Consumption Issues for Environmentalists. $A$ literature search and bibliography prepared by the Population Reference Bureau for the Pew Charitable Trusts, Global Stewardship Initiative, Washington, D. C. (cuadernillo).

Ehrlich, Paul y J. Holdren (1971), "The Impact of Population Growth", Science, núm. 171, pp. 1212-1217.

(1974), "Human Population and the Global Environment", American Scientist, núm. 62, pp. 282-292.

Engelman, Robert (1994), Stabilizing the Atmosphere: Population, Consumption and Greenbouse Gases, Washington; D. C., Population and Environment Program-Population Action International.

García, Rolando (1986), "Conceptos básicos para el estudio de sistemas complejos", en E. Leff (coord.), Los problemas del conocimiento y la perspectiva ambiental del desarrollo, México, Siglo XXI.

García, Brígida, Humberto Muñoz y Orlandina de Oliveira (1982), Hogares y trabajadores en la ciudad de México, México, El Colegio de México-UNAM.

Economía, Sociedad y Territorio, vol. I núm. 2, 1997, 225-256

Heilig, Gerhard (1993), How Many People Can Be Fed on Earth?, Laxenburg, Austria, IIASA, Working Paper WP-93-40.

(1995), Lifestyles and Global Land-use Change: Data and Thesis, Laxenburg, Austria, MASA, Working Paper WP-95-91, septiembre.

Hogan, Daniel (1993), "Capacidad de carga poblacional: Rehabilitando un concepto", en Haydea Izazola y Susana Lerner (comps.), Población y ambiente: inuevas interrogantes a viejos problemas?, México, SOMEDE-El Colegio- de México-The Population Council

1995), "Population, Poverty and Pollution in Cubatao, Sao Paulo", en Alina Potrikowska y John Clarke (eds.) Population and Environment in Industrialized Regions, Número Especial, 64, Geographia Polonica, Varsovia, Academia Polaca de Ciencias-Instituto de Geografía y Organización Espacial, pp. 201-224.

Izazola, Haydea y Catherine Marquette (1995), "Migration in Response to the Urban Environment: Out-migration by Middle Class Women and their Families from Mexico City after 1985", en Alina Potrikowska y John Clarke (eds.), Population and Environment in Industrialized Regions, Número Especial, 64, Geograpbia Polonica, Varsovia, Academia Polaca de Ciencias-Instituto de Geografía y Organización Espacial, pp. 225-256.

y Susana Lerner (comps.) (1993), Población y ambiente: inuevas interrogantes a viejos problemas?, México, SOMEDE-E1 Colegio de México-The Population Council.

Landa, Rosalva y Julia Carabias (1997), "Deterioro ambiental, una propuesta conceptual para zonas rurales de México" (en este volumen).

Leff, Enrique (1993), "La interdisciplinariedad en las relaciones población-ambiente. Hacia un paradigma de demografía ambiental", en Haydea Izazola y Susana Lerner (comps.), Población y ambiente: inuevas interrogantes a viejos 
probtemas?, México, SOMEDE-El Colegio de México-The Population Council.

(1995), “De quién es la naturaleza? Sobre la apropiación social de los recursos naturales", Gaceta Ecológica, núm. 37, México, Instituto Nacional de Ecología-Secretaría del Medio Ambiente, Recursos Naturales y Pesca, pp. 58-64.

Lerner, Susana y André Quesnel (1982), "Una familia como categoría analítica en los estudios de población. Propuesta de un esquema de análisis", en CONACYT, Memorias de la Segunda Reunión Nacional sobre la Investigación Demográfica en México, México, CONACYT.

Marquette, Catherine (1995), Housebolds Characteristics and Deforestation: A Study on the Northern Ecuadorian Amazon Frontier, Nueva York, Departamento de Sociología/Universidad de Fordham, tesis de doctorado en demografía.

y Richard Bilsborrow (1994), "Population and the Environment in Developing Countries: Literature Survey and Research Bibliography", borrador, Nueva York, ONU, Population Division of the Economic and Social Information and Policy Analysis.

Martine, George (1993), "Población, crecimiento y modelo de civilización: dilemas ambientales del desarrollo”, en Haydea Izazola y Susana Lerner (comps.), Población y ambiente: inuevas interrogantes a viejos problemas?, México, SOMEDE-El Colegio de México-The Population Council. - .

Martínez, Carolina y L. Vargas (en prensa), "Ambiente y salud en Xochimilco: una aproximación cualitativa", Estudios de Antropología Biológica, vol. VIII, México, IIA-UNAM.

Mc Nicoll, Geoffrey (1994), "Mediating Factors Linking Population and the Environment", en ONU, Population, Environment and Development: Proceedings of the United Expert Group Meeting on Population, Environment and Dewelopment, Nueva York, ONU.
Meggers, Betty (1976), Amazonia. Hombre y cultura en un paraíso ilusorio, México, Siglo XXI.

Mertens, Walter et al. (1982), Reflexiones teórico-metodológicas sobre investigacionesen población, México, El Colegio de México.

Organización de las Naciones Unidas (ONU) (1994), Population, Environment and Development: Proceedings of the United Expert Group Meeting on Population, Environment and Development, Nueva York, ONU.

(1996), Población Mundial 1996, Nueva York, ONU.

Pichón, Francisco (en preñssa), “Agricultural Settlement, Land Use and Deforestation in the Ecuadorian Amazon Frontier: A Micro-Level Analysis of Colonists' Land Allocation Behavior", en Barbara Badaut y William Moomaw (eds.), Population/Environment Equation: Implications for Future Security, Londres, MacMillan.

Pimentel, David (1997), "Livestock Production: Energy Inputs and the Environment", ponencia presentada en la Reunión de la Sociedad Canadiense de Ciencias Animales, Montreal, Canadá, 24 al 26 de julio.

Provencio, Enrique (s/f), "Patrón de consumo, desigualdad y sustentabilidad: Algunas consideraciones generales y para el caso de México", Programa de Estudios Avanzados en DesarrolloSustentable y Medio Ambiente(LEAD-México), El Colegio de México (mimeo.).

Schmink, Marianne y Charles Wood (1992), Contested Frontiers in Amazonia, Nueva York, Columbia University Press.

Schteingart, Martha (coord.) (1997), Pobreza, condiciones de vida y salud en la ciudad de México, México, El Colegio de México.

Sydenstrycker Neto, John y Stephen A. Vosti (1993), "Household Size, Sex Composition and Land Use in Tropical Moist Forest: Evidence from the Machadinho Colonization Project, Rondônia, Brazil", ponencia presentada en la 